\title{
MUSINGS
}

\section{From personalized to public health genomics}

\author{
Greg Gibson ${ }^{1 *}$ and Peter M Visscher ${ }^{2}$
}

It is a sign of the times that most of the buzz around genomic medicine has more to do with personalized medicine than public health [1]. This is perhaps consistent with the prevailing economic sentiment in developed countries that benefits accruing to the few gradually transfer to the majority. Healthy societies also take steps to ensure that there is equitable distribution of wealth, which should be construed to include much more than financial wellbeing. We can thus ask to what extent genomics can be used to improve the prospects of as many people as possible.

For perspective, consider the public health benefits that are generally considered to have had the greatest impact globally. Rather than individualized treatments, these are things like access to fresh water, closed sewage systems, better knowledge of hygiene, widespread antibiotic usage, mosquito nets, and education of women. More recently, recording patients' family medical history, the adoption of electronic medical records, and the trend toward standardization of medical care on the basis of evidence of efficacy are all set to benefit a large number of people, at least in developed countries. What are the genomic equivalents?

The poster child for successful personalized genomics is the case of the Beery twins [2]. Diagnosed early in life with DOPA-responsive dystonia, but incompletely responsive to dopamine therapy, the discovery of compound heterozygous mutations in their sepiapterin reductase gene led to supplementation with the serotonin precursor 5-hydroxytryptophan, which turned the twins' lives around. Early adopters of whole-exome sequencing in the context of congenital pediatric defects, including ciliopathies, craniofacial abnormalities, primary immune deficiencies and developmental delay, report impressive levels of success in identifying causal mutations, bringing peace of mind to parents and in some cases suggesting new medical interventions. And cancer treatment is already being reshaped by the deep sequencing of tumor genomes and transcriptomes, with the objective of

${ }^{*}$ Correspondence: greg.gibson@biology.gatech.edu

'School of Biology, Georgia Institute of Technology, Atlanta, GA 30332, USA

Full list of author information is available at the end of the article identifying the most suitable chemotherapeutic targets in refractory cases [3]. Extraordinary and transformative as these achievements are, it is hard to imagine them directly benefiting more than a minority - perhaps $5 \%$ - of the population. However, we can see five ways in which personal genomics may generalize to public health genomics.

(1) Trickle-down genomics. No doubt the most likely development arising from personal genomics will be a gradual expansion of the number of people whose personal genomes become a part of their individualized treatment. The next frontier may be genomic risk assessment for adult-onset congenital decline, but we will also see the extension of health benefits from probands to family members. In this context, it is worth highlighting a recent report from 23andMe [4] that the discovery of $B R C A 1$ or $B R C A 2$ mutations in 32 people led to the detection of the mutations in 13 of 30 relatives of these people who chose follow-up evaluation, with an overall conversion of initial anxiety into positive outcomes.

(2) Biological knowledge. In their review of the first five years of genome-wide association studies (GWAS), Visscher et al. [5] countered the perception in some circles that common variant analysis has had little utility because it is rarely clinically actionable, by observing the gains in knowledge of new biology have been unprecedented. It is doubtful whether many scientists, if they had been told 10 years ago that the investment of half a billion dollars would lead to the discovery of over 100 independent loci for each of the common diseases that afflict humans, would have baulked at the expenditure. The discoveries have highlighted new cellular and molecular mechanisms, informed us about the architecture of disease, and in time will surely translate into new medical care options for diseases that collectively afflict more than half the population.

(3) Wellness genomics. Genomic profiling can already facilitate personal risk assessment not just for people with overt disease, but also for healthy individuals, particularly middle-aged adults as they begin to deal with medical issues. Some will age gracefully, but most will encounter pain and loss of organ function that will severely constrain their lifestyle, so one objective for personalized medicine should be helping people to make better health decisions [6]. This is in fact one of the 
motivations for medical genomic profiling companies, to promote self-knowledge and thereby change the culture from one of 'doctor, I'm sick, make me better' to one of 'the more informed I am, the better decisions I can make for myself'. Society also has an interest in shifting the burden from government programs to individual responsibility. Genetics alone will never be accurately predictive for most conditions, and many challenges remain in the interpretation and communication of information between patients and physicians [7]. However, combined with functional genomic, environmental and clinical profiles, genomic profiling has the potential to classify elevated risk, which may be useful for each one of us.

(4) Genomics of disease progression. So far, both GWAS and genome sequencing have focused on the genetic basis of the onset of disease and its causes. Yet clinically, arguably the more important need for personal profiling is in understanding how disease progresses: why do some patients go into remission whereas others constantly battle flares and relapses? Pain and depression are equally important aspects of patient experience, yet have received little attention from genomicists. On this note, it is striking that the number one source of morbidity, lower back pain, is completely absent from the GWAS catalog, despite at least anecdotal heritability [8]. Relatedly, there are a growing number of pharmacogenetic tests to assess personal response to drugs, and there is also ample room for assessing response to other types of interventions, including dietary change and medical devices.

(5) Global health genomics. Given the epidemiological transition globally from infectious and nutritional disease to the Western profile of metabolic, immunological, cognitive, and age-related diseases [9], there seems to be a moral obligation to ensure that advances in the developed world are quickly transferred to developing countries. Cost and sheer numbers, not to mention priorities and infrastructure, mean that personal genomics will only be accessible to a tiny minority for the near future, underscoring the imperative to ensure that the benefits of medical genomics are transferred through indirect mechanisms. First-rate genome research institutions are emerging all over the world, and international networks connect researchers globally, so the conceptual challenge is how to partner traditional medical programs with genome-oriented public health programs [10]. We envisage, for example, initiatives that use genomics to characterize the identity and physiological impact of environmental toxins, to enhance newborn screening directed at early interventions, and to target scarce medical resources to the most at risk individuals and those most likely to respond to treatment.

Contemporary human genetics faces a tension between discovery genetics, performed most efficiently at the scale of $N=100,000$, and translational medical genetics, performed necessarily at $N=1$. As we learn how to read individual genomes, it is natural that attention will focus initially on large-effect rare variants, as these are considered to be most likely to define clinical targets. Yet basic quantitative genetics tells us that the reasons why brothers resemble sisters, and why populations differ in disease prevalence, have more to do with the genetic polymorphisms we share than the mutations we own privately. Harnessing this insight, in ways we can only begin to perceive, has the potential to achieve substantial economic and public health efficiencies. Because our genetic and environmental backgrounds remain essential components of risk, familiarity with the common genome is one key to ensuring that genomics becomes a public health enterprise.

\section{Abbreviations}

GWAS, genome-wide association study.

\section{Competing interests}

The authors declare that they have no competing interests.

\section{Author details}

'School of Biology, Georgia Institute of Technology, Atlanta, GA 30332, USA. ${ }^{2}$ Queensland Brain Institute and University of Queensland Diamantina Institute, Brisbane 4072, Australia.

Published: 19 July 2013

\section{References}

1. Hamburg MA, Collins FS: The path to personalized medicine. N Engl J Med 2010, 363:301-304.

2. Bainbridge MN, Wiszniewski W, Murdock DR, Friedman J, Gonzaga-Jauregui C, Newsham I, Reid JG, Fink JK, Morgan MB, Gingras MC, Muzny DM, Hoang LD, Yousaf S, Lupski JR, Gibbs RA: Whole-genome sequencing for optimized patient management. Sci Transl Med 2011, 3:87re3.

3. Roychowdhury S, lyer MK, Robinson DR, Lonigro RJ, Wu YM, Cao X, KalyanaSundaram S, Sam L, Balbin OA, Quist MJ, Barrette T, Everett J, Siddiqui J, Kunju LP, Navone N, Araujo JC, Troncoso P, Logothetis CJ, Innis JW, Smith DC, Lao CD, Kim SY, Roberts JS, Gruber SB, Pienta KJ, Talpaz M, Chinnaiyan AM: Personalized oncology through integrative high-throughput sequencing: a pilot study. Sci Transl Med 2011, 3:111ra121.

4. Francke U, Dijamco C, Kiefer AK, Eriksson N, Moiseff B, Tung JY, Mountain JL: Dealing with the unexpected: consumer responses to direct-access $B R C A$ mutation testing. Peer J 2013, 1:e8.

5. Visscher PM, Brown MA, McCarthy MI, Yang J: Five years of GWAS discovery. Am J Hum Genet 2012, 90:7-24.

6. Patel CJ, Sivadas A, Tabassum R, Preeprem T, Zhao J, Arafat D, Chen R, Morgan AA, Martin GS, Brigham KL, Butte AJ, Gibson G: Whole genome sequencing in support of wellness and health maintenance. Genome Med 2013, 5:58.

7. Chute CG, Kohane IS: Genomic medicine, health information technology, and patient care. JAMA 2013, 309:1467-1468.

8. Battié MC, Videman T, Levalahti E, Gill K, Kaprio J: Heritability of low back pain and the role of disc degeneration. Pain 2007, 131:272-280.

9. Murray CJL, Lopez AD: Global mortality, disability, and the contribution of risk factors: Global Burden of Disease Study. Lancet 1997, 349:1436-1442.

10. Burke W, Khoury MJ, Stewart A, Zimmern R; for the Bellagio Group: The path from genome-based research to population health: development of an international public health genomics network. Genet Med 2006, 8:451-458.

doi:10.1186/gm464

Cite this article as: Gibson G, Visscher PM: From personalized to public health genomics. Genome Medicine 2013, 5:60. 